\title{
Cochrane Systematic Review Antiviral Treatment for Bell's Palsy: an Opposing Opinion!
}

\author{
Ahmed B. Bayoumy ${ }^{1}$ (D) J. Alexander de Ru ${ }^{1}$
}

Accepted: 28 May 2020 / Published online: 14 June 2020

(C) The Author(s) 2020

\begin{abstract}
This articles discusses the recently published Cochrane systematic review (CSR) on antiviral treatment for Bell's palsy. The CSR concluded that there was no clear difference in rates of incomplete recovery between combination therapy and corticosteroids alone. The conclusions of this CSR are in our opinion misleading, illogical, and based on an overly restrictive view on the use of evidence. This article shows that the majority of studies are in favor of the combination therapy in regard to recovery and reduction of long-term sequelae. Based on all available evidence, the combination of antivirals and corticosteroids seems to be a useful treatment option and should be discussed with patients by shared decision-making.
\end{abstract}

Keywords Bell's palsy $\cdot$ Antiviral therapy $\cdot$ Acyclovir $\cdot$ Famciclovir $\cdot$ Valaciclovir $\cdot$ Corticosteroids $\cdot$ Cochrane systematic review

\section{Introduction}

Recently, the Cochrane systematic review (CSR) on antiviral treatment for Bell's palsy was updated [1]. The conclusions of this CSR are in our opinion misleading, illogical, and based on an overly restrictive view on the use of evidence.

Suppose a patient has a disease that may lead to severe morbidity with a chance of everlasting deficits in $16 \%$ of patients. Suppose that the medication for this disease under review costs only 156 GBP (175 euro, 205 USD) at most and that addition of this medication has no more side effects than the standard therapy alone. Now suppose that this therapy has an overall risk ratio of $0.54(0.38-0.77)$ as demonstrated in the forest plot of Fig. 4 in the CSR (see Fig. 1) [1]. Can anyone imagine withholding such a therapy from patients?

Our conclusions that will be supported with our reasoning and statistical analysis are as follows:

- Antivirals in combination with steroids are superior than steroids alone in the treatment of Bell's palsy;

This article is part of the Topical Collection on Medicine

Ahmed B. Bayoumy

a.b.bayoumy@amsterdamumc.nl

1 Department of Otolaryngology, Ministry of Defense, Central Military Hospital, Lundlaan 1, 3584 EZ Utrecht, The Netherlands
- An overly restrictive view on evidence is not helpful in decision-making;

- The CSR is probably written with too much author bias.

Bell's palsy is an acute, unilateral paralysis or weakness of facial musculature consistent with peripheral facial nerve dysfunction, of no detectable cause [1].

Only $71 \%$ of patients with Bell's palsy recover completely without therapy. Thirteen percent ends up with only mild deficits, but the remaining $16 \%$ have permanently diminished function. Synkinesis and crocodile tears are the sequelae of inappropriate recovery of the nerve following severe paresis/ paralysis [2].

The cause of Bell's palsy is still debated, and it has been thought that viral infections (herpes simplex and herpes zoster viruses) may cause Bell's palsy. The viral hypothesis has gained much support in the last couple of years, since the isolation of the herpes simplex virus type 1 genome from the saliva and facial nerve fluid from patients with this condition [3-5]. Scientific progress made it possible to demonstrate these viral infections, something that was not possible more than two centuries ago, when Sir Charles Bell described the "idiopathic" paralysis.

The pathophysiological mechanism responsible for Bell's palsy is that viral infection causes local inflammation of the facial nerve. Due to this inflammation, the nerve needs to expand. But, there is simply no space within the facial canal for a swollen facial nerve so the nerve compresses its own 
Risk Ratio

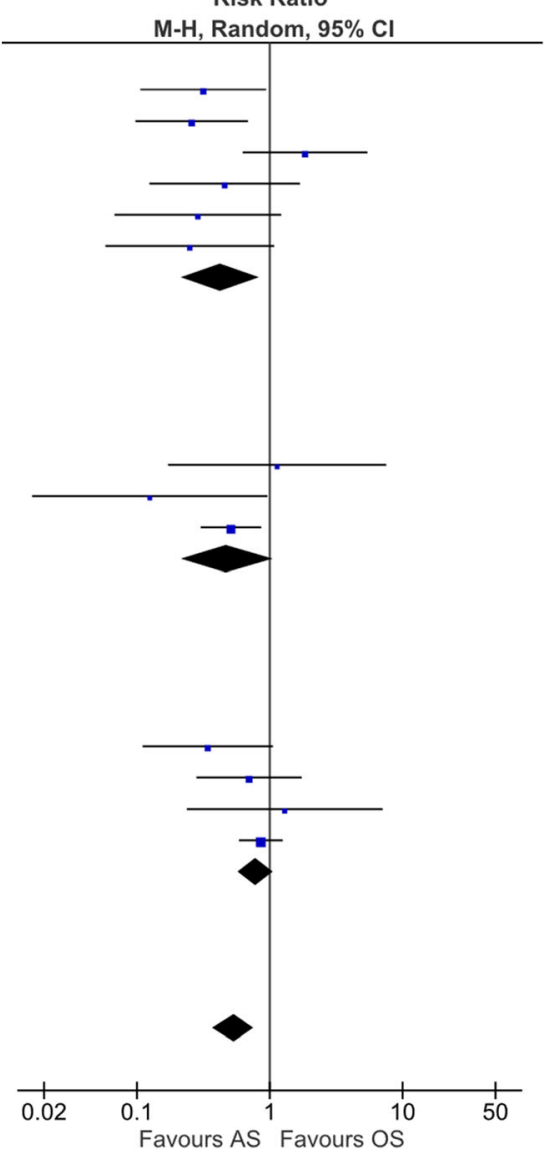

Fig. 1 The overall forest plot of antivirals plus corticosteroids versus corticosteroids plus placebo or no treatment. The outcome was incomplete recovery at the end of study as based on the CSR [1]

vascular circulation. It is therefore crucial to start immediate treatment to reduce inflammation and edema around the facial nerve [6]. So, it is generally accepted to use corticosteroids for treatment of Bell's palsy; this was also confirmed by the CSR [7]. However, several authors did not have consentient conclusions regarding the superiority of corticosteroids and antiviral combination therapy over corticosteroid monotherapy [8].

\section{Main Text}

According to the abstract of the CSR, corticosteroids are widely used, but the effectiveness of additional treatment with an antiviral agent is uncertain. Therefore, the main research question was to determine the effect of adding antivirals to corticosteroids. The plain language summary states that the focus in this updated review was combined therapy with antiviral drugs and corticosteroids, as there is already good evidence that corticosteroids reduce rates of incomplete recovery [1].
However, the CSR changes its research question and in the "Objectives" in the abstract, it becomes clear that the CSR is, again, also assessing the effects of antivirals alone. This, in our opinion, is a non-relevant research question. Based on the pathophysiology, reducing swelling is the most important factor. Obviously, corticosteroids will perform better than antivirals. Therefore, the answer to this altered question creates a fog that hides the answer to the primary research question, a distraction that should have been accounted for beforehand and therefore abandoned! Likewise, the CSR concludes, in case of motor synkinesis or crocodile tears, that there was no clear difference in this outcome with antivirals alone compared with a placebo. So, this CSR view frames the outcome in a negative direction by presenting outcomes that were not relevant clinical issues. However, we should be seeking approximate answers to the right questions, not exact answers to the wrong ones [9].

The main "Results" section of the abstract states that fourteen trials including 2488 participants met the inclusion criteria. The "Methods" section states "unlike the previous version of the review we did not exclude studies at high risk of bias." A very promising start indeed.

Unfortunately, the plain language summary describes the main problem of this review: the authors chose to base their conclusions only on data from three studies at a lower risk of bias.

Although the reasons for exclusion are written down perfectly according to the "standard Cochrane procedures," we are of the opinion that it would have been wise to search the total body of evidence. Sir Austin Bradford Hill wrote "I would myself put a good deal of weight upon similar results reached in quite different ways, e.g. prospectively and retrospectively." Because of the claimed uncertainty about the evidence, the authors would indeed have better based their conclusion on all available studies. To us it is puzzling that the authors did not take into account the larger amount of studies for their conclusions. Studies were removed because of possible bias due to epidemiological flaws; however, the probability of bias caused by a yet unknown factor in the three studies alone is likely to be higher than any bias caused by epidemiological flaws in the excluded studies together.

Before we dive deeper in the numbers, we would like to share our thoughts on the methodology of most studies. Many authors used the classical null hypothesis of no effect. However, this is not ideal because there is reasonable chance that antivirals can contribute to the improvement of a disease caused by a virus. Thus, taking a Bayesian a priori chance into account would be a more appropriate option. However, this kind of statistics is difficult for most physicians. 
Furthermore, there is also a problem to demonstrate a genuine effect because most patients recover spontaneously or because of the therapy with corticosteroids. So, the extra benefits from antiviral therapy are therefore concealed in most patients. If one considers that the most severely affected patients will get residual complications, it would be logical to concentrate on these patients.

So, let us consider the most hindering complications of Bell's palsy (i.e., motor synkinesis or crocodile tears) and ask ourselves the question if we could reduce these by adding antivirals to corticosteroid therapy. For this outcome, the CSR states: "that fewer participants experienced these long-term sequelae after antivirals plus corticosteroids than after corticosteroids plus placebo or corticosteroids alone (RR 0.56, 95\% CI 0.36 to $0.87 ; 2$ trials, $\mathrm{N}=469$ )." Thus, if we would rationally consider prescribing the combination therapy for our patients, then the answer based on this result already should be: Yes!

In our opinion, combination therapy should especially be recommended for patients with higher probability of poor recovery (i.e., elderly and patients with initially severe paralysis).

The next step would be to look if those patients with severe paralysis actually benefit from the combination therapy. Unfortunately, the CSR restricted the main analysis to only two studies. They conclude that the proportion of severe cases with incomplete recovery at 6 months showed no clear effect in the combination therapy group versus the steroids alone or no treatment group (RR $0.82,95 \%$ CI 0.57 to 1.17 ) [1]. Though this might not be "statistically significant" because the $\mathrm{CI}$ is also extending beyond 1 , the result can be very much in line with our clinical assumption that RR 0.82 might be close to the real effect; so clinical relevance is high. Especially, in relation to the previous point showing reduction of severe sequelae, this outcome fits - of course in this case the opposite is true because we are discussing reduction of morbidity! — what Anderson recently described as: "suppose it represented an association between a food additive and cancer in laboratory animals; although we would not reject the hypothesis that there is no relationship, it would be difficult to find a statistician willing to consume the substance" [10], or as Amrhein et al. wrote: "inferences should be scientific, and that goes far beyond the merely statistical. Factors such as background evidence, study design, data quality and understanding of underlying mechanisms are often more important than statistical measures such as P values or intervals" [11].

When the analysis used all the available evidences, the proportion of severe cases with incomplete recovery was slightly lower in the AS (combination therapy) group versus that in the OS (steroids alone or steroids plus placebo) group ( $17 \%$ vs $29 \%$ in people with severe Bell's palsy; RR 0.64, $95 \%$ CI 0.41 to 0.99 ) [1]. If we would frame the data in terms of recovery, then $83 \%$ of severe patients in the AS group and
$71 \%$ in the OS group would recover from Bell's palsy. So, when taking into account all evidences, we get a clinical very relevant positive result and narrowing of the CI. Again, it actually is what we expected. So, yes we have a good treatment option for patients with severe Bell's palsy! Alas, however inconceivable, the CSR calls this result imprecise.

For the question if combination therapy is superior including all categories of paresis, at first the CSR is describing the results of only three studies. Still the RR shows a point estimate of RR 0.81 (CI 0.38-1.74) that could concur with a hypothesis of superiority of combination therapy. That is completely in line with the outcome of the severe cases only, if it were not for the CI being large. Let us consider the full data set from Fig. 4 in the CSR [1]. This shows a crystal clear relevant effect RR 0.54 (CI 0.38-0.77). In total, 101 of 872 (12\%) patients in the AS group and 170 of 857 (20\%) patients in the OS group had incomplete recovery. The lower RR combined with the $8 \%$ difference in recovery in favor of the AS group demonstrates a meaningful reduction of morbidity with combination therapy.

If we add information from the four additional studies that were excluded by the CSR and not even included in their Fig. 4, then we see again that the results become even more in favor of the combination therapy RR 0.52 (CI $0.39-0.68)$ [1]. Notice that not only the point estimate is more in favor, but that the confidence interval is narrowing as well (see Fig. 2).

Furthermore, if we do the same analysis but for complete recovery using the same studies as above, we obtain RR 1.10 (CI 1.04-1.16, 17 studies, $N=4403$ ) in favor of the combination therapy group. In total, 1751 out of $1958(89.4 \%)$ had complete recovery (House-Brackmann scale (HB) $\leq 2$ or $\mathrm{HB}=1$ ) in the combination therapy group, whereas 2061 out of 2445 (84.3\%) had complete recovery in the corticosteroids and placebo or no treatment group. Thus, when considering complete recovery, it is also in favor of the combination therapy.

As shown in Fig. 3 of this article, it is notable that the results are consequently in favor of the combination therapy for different antiviral agents. Only three of 17 studies are around or above a risk ratio of 1.0. The remaining 14 studies are all under the risk ratio of 1.0 ; therefore, most studies present outcomes in favor of the combination therapy.

The Minnerop article describes a very relevant positive effect for combination therapy compared with corticosteroids alone: more patients showing some degree of recovery, the patients with the greatest number of recovery points (on the House-Brackmann scale), and fewer patients remaining at the worse HB IV and V scores [12].

The latest Cochrane study describes the fact that the data of the Minnerop study might not be as positive for the combination therapy group as previously expected. The CSR authors got unpublished information that shows that, somewhere 
Fig. 2 The forest plot of antivirals plus corticosteroids versus corticosteroids plus placebo or no treatment. The outcome was incomplete recovery at the end of study with additional studies that were excluded from the CSR

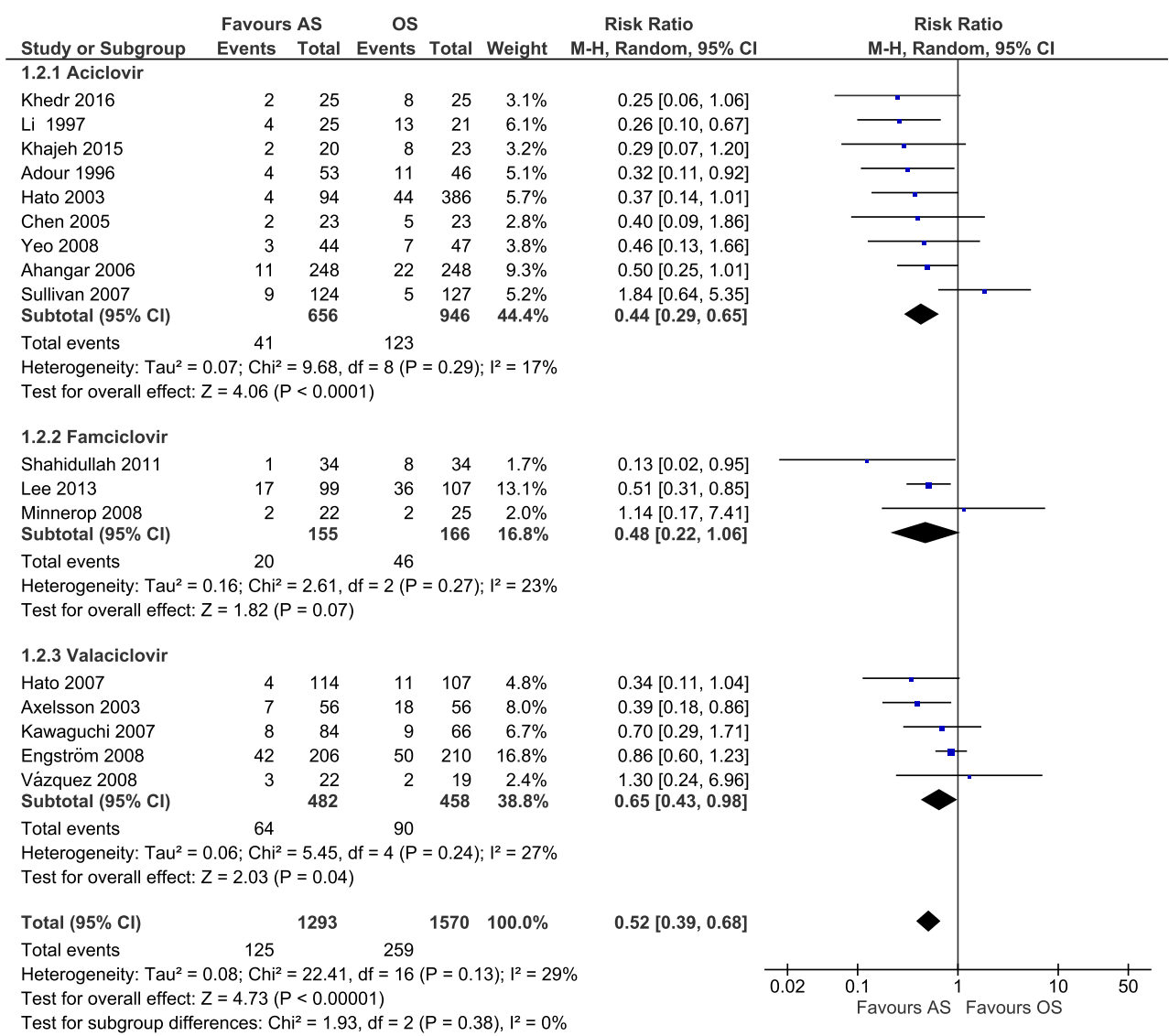

along the line, the data, apparently, were not that positive. The CSR presentation does not show the Minnerop data, but only provides us with a summary and "interpretation" by the CSR authors.

Both authors of this article have separately and on multiple occasions tried to get in contact with Minnerop. Unfortunately, we did not get any response. Within the Royal Netherlands

Fig. 3 Funnel plot of the three antiviral therapies; the risk ratios are for incomplete recovery calculated by comparing antivirals plus corticosteroids versus corticosteroids plus placebo or no treatment. The black circles are acyclovir, the red diamond are famciclovir, and the green squares are valaciclovir
Marine Corps, it is taught that trust is good, but that control is better. So, until Minnerop publishes new data and a new conclusion, we prefer to stick with their previous published data instead of "trusting" the CSR authors that the provided calculations are correct.

The CSR made a sensitivity analysis to investigate the effect of removing studies with a follow-up of less than 6 months. This

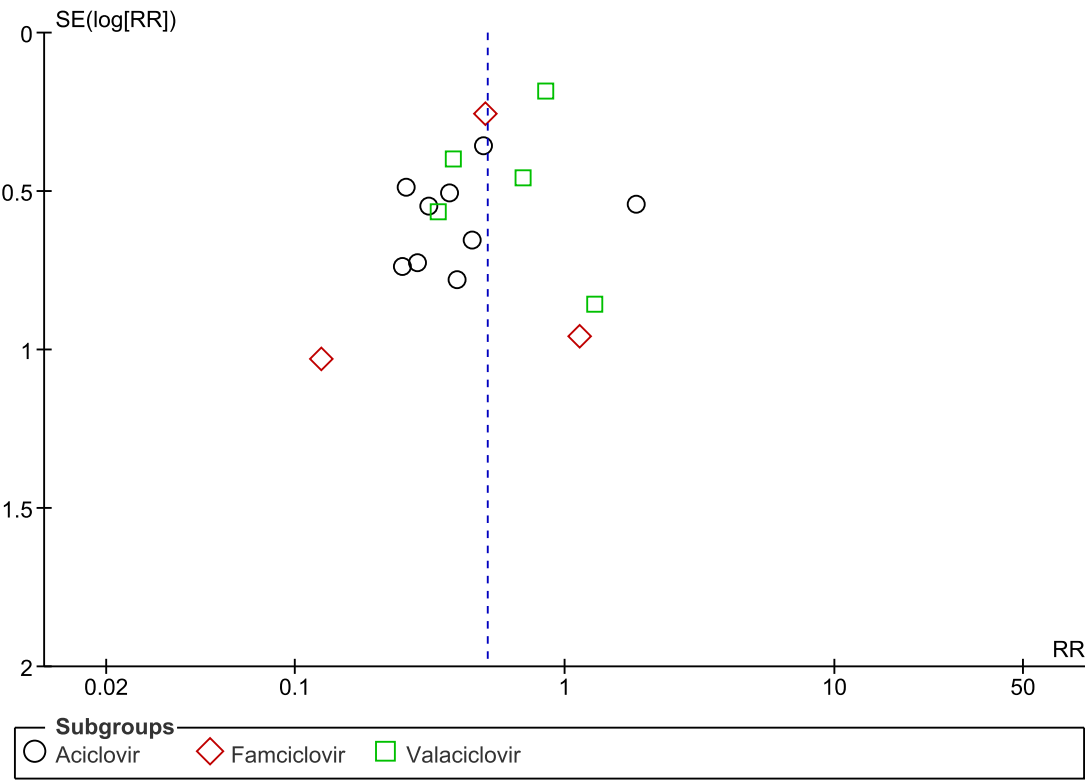


did not substantially alter the findings. We performed a sensitivity analysis based on the "odd-ones-out" principle removing the most positive and the most negative study (Sullivan and Shadidullah) $[8,13]$. Removal of the "odd-ones" out of Fig. 2 causes a remarkable narrowing of the CI (RR 0.53; CI 0.42$0.66)$. Moreover, the heterogeneity between the studies became remarkably low.

Furthermore, we observe that the most "negative" contributing study in this CSR is that of Sullivan et al. [8]. Herein, a total of $7 \%$ of all patients with an initial low-grade paresis (House-Brackmann grade I) were included. Thus, these patients were already fully recovered at inclusion. Furthermore, the dosage of antivirals used in this study was also too small to treat a possible herpes zoster infection. It is the only study in which combination therapy appears to worsen outcomes. It was suggested by the authors that the Jarisch-Herxheimer reaction might account for this strange outcome [14]. Such outcome has not been described before with this combination therapy and it has not been mentioned in any of the other studies.

Finally, we ask ourselves if the CSR was completely free of author bias. The fact is that several authors of the Sullivan study have also written this CSR; so authors of the study that contributes the most to the negative results in this CSR are writing the negatively framed overall conclusion. We therefore get the idea that the conclusions of the CSR might be biased.

\section{Conclusion}

The recent Cochrane systematic review concluded that there was no clear difference in rates of incomplete recovery between combination therapy and corticosteroids alone. This article shows that the majority of studies are in favor of the combination therapy in regard to recovery and reduction of long-term sequelae. Based on all available evidence, the combination of antivirals and corticosteroids seems to be a useful treatment option and should be discussed with patients by shared decision-making.

\section{Compliance with Ethical Standards}

Conflict of Interest The authors declare that they have no conflict of interest.

Ethical Approval No ethical approval was necessary.

Informed Consent No informed consent was necessary.

Open Access This article is licensed under a Creative Commons Attribution 4.0 International License, which permits use, sharing, adaptation, distribution and reproduction in any medium or format, as long as you give appropriate credit to the original author(s) and the source, provide a link to the Creative Commons licence, and indicate if changes were made. The images or other third party material in this article are included in the article's Creative Commons licence, unless indicated otherwise in a credit line to the material. If material is not included in the article's Creative Commons licence and your intended use is not permitted by statutory regulation or exceeds the permitted use, you will need to obtain permission directly from the copyright holder. To view a copy of this licence, visit http://creativecommons.org/licenses/by/4.0/.

\section{References}

1. Gagyor I, Madhok VB, Daly F, Sullivan F. Antiviral treatment for Bell's palsy (idiopathic facial paralysis). Cochrane Database Syst Rev. 2019;9:Cd001869.

2. Holland NJ, Bernstein JM. Bell's palsy. BMJ Clin Evid. 2014;2014:1204.

3. Adour KK, Bell DN, Hilsinger RL Jr. Herpes simplex virus in idiopathic facial paralysis (Bell palsy). JAMA. 1975;233(6):52730 .

4. Murakami S, Mizobuchi M, Nakashiro Y, Doi T, Hato N, Yanagihara N. Bell palsy and herpes simplex virus: identification of viral DNA in endoneurial fluid and muscle. Ann Intern Med. 1996;124(1 Pt 1):27-30.

5. Peitersen E. Bell's palsy: the spontaneous course of 2,500 peripheral facial nerve palsies of different etiologies. Acta Otolaryngol Suppl. 2002;549:4-30.

6. Liston SL, Kleid MS. Histopathology of Bell's palsy. Laryngoscope. 1989;99(1):23-6.

7. Gronseth GS, Paduga R. Evidence-based guideline update: steroids and antivirals for Bell palsy: report of the Guideline Development Subcommittee of the American Academy of Neurology. Neurology. 2012;79(22):2209-13.

8. Sullivan FM, Swan IR, Donnan PT, et al. Early treatment with prednisolone or acyclovir in Bell's palsy. N Engl J Med. 2007;357(16):1598-607.

9. Tong C. Statistical inference enables bad science; statistical thinking enables good science. Am Stat. 2019;73(sup1):246-61.

10. Anderson AA. Assessing statistical results: magnitude, precision, and model uncertainty. Am Stat. 2019;73(sup1):118-21.

11. Amrhein V, Greenland S, McShane B. Scientists rise up against statistical significance. Nature. 2019;567(7748):305-7.

12. Minnerop M, Herbst M, Fimmers R, Kaabar P, Matz B, Klockgether T, et al. Bell's palsy: combined treatment of famciclovir and prednisone is superior to prednisone alone. $\mathrm{J}$ Neurol. 2008;255(11):1726-30.

13. Shahidullah M, Haque A, Islam MR, Rizvi AN, Sultana N, Mia BA, et al. Comparative study between combination of famciclovir and prednisolone with prednisolone alone in acute Bell's palsy. Mymensingh Med J. 2011;20(4):605-13.

14. Lockhart P, Daly F, Pitkethly M, Comerford N, Sullivan F. Antiviral treatment for Bell's palsy (idiopathic facial paralysis). Cochrane Database Syst Rev. 2009;(4):CD001869. https://doi. org/10.1002/14651858.CD001869.pub4.

Publisher's Note Springer Nature remains neutral with regard to jurisdictional claims in published maps and institutional affiliations. 